\title{
Prevalência de hepatozoonose canina no município de Areia, Paraíba, Brasil
}

\author{
Maria das Graças da Silva Bernardino * \\ Maria Vanuza Nunes de Meireles \\ Edijanio Galdino da Silva \\ Fábio Júnior Rodrigues Xavier \\ Fabiana Satake \\ Laboratório de Patologia Clínica Veterinária, Departamento de Ciências Veterinárias \\ Universidade Federal da Paraíba, Centro de Ciências Agrárias \\ Cidade Universitária, s/n, CEP 58.397-000, Areia - PB, Brasil \\ * Autor para correspondência \\ maryangel_ufpb@hotmail.com
}

Submetido em 19/07/2015

Aceito para publicação em 14/11/2015

\section{Resumo}

A hepatozoonose canina é uma doença parasitária de caráter debilitante, imunossupressora e de difícil diagnóstico clínico. A prevalência da doença em cães já foi descrita em vários estados brasileiros, todavia na região Nordeste os estudos ainda são escassos. Em virtude disso, o trabalho objetivou determinar a prevalência da hepatozoonose canina no município de Areia, Paraíba, Brasil. Os cães foram examinados clinicamente e foram colhidas amostras de sangue circulante e periférico (ponta de orelha). Das 151 amostras analisadas, 9,3\% dos cães foram positivos para Hepatozoon canis, a partir da visualização de gamontes parasitando neutrófilos. $\mathrm{O}$ grau de parasitemia variou de leve a intenso. As alterações hematológicas observadas foram diferentes entre os animais, não apresentando padrão de resposta à infecção. A forma subclínica foi observada em $50 \%$ dos cães e os demais apresentaram sinais clínicos inespecíficos. Conclui-se que há a ocorrência da hepatozoonose em cães da área urbana do município. Além disso, os cães infectados com H. canis apresentaram alterações hematológicas e sinais clínicos inespecíficos.

Palavras-chave: Epidemiologia; Hematologia; Hemoparasita; Hepatozoon canis

\section{Abstract}

Prevalence of canine hepatozoonosis in Areia, Paraíba, Brazil. Canine hepatozoonosis is a parasitic disease of debilitating nature. Also, it is immunosuppressive and difficult to diagnose clinically. The prevalence of the disease in dogs has been described in several Brazilian states, but studies are still scarce in the Northeast region. Accordingly, this study aimed to determine the prevalence of canine hepatozoonosis in Areia, Paraíba, Brazil. The dogs were clinically examined, and peripheral blood samples were obtained from the tip of the ear. Of the 151 dogs analyzed, 9.3\% were positive for Hepatozoon canis by the demonstration of gamonts parasitizing neutrophils. The degree of parasitemia ranged from mild to intense. The observed hematological changes were different between animals and showed no pattern of response to infection. The subclinical form 
was observed in $50 \%$ of dogs and the others had nonspecific clinical signs. We conclude that hepatozoonosis occurs in dogs in the urban area of the city studied and that dogs infected with $H$. canis show hematological changes and nonspecific clinical signs.

Key words: Epidemiology; Hematology; Hemoparasite; Hepatozoon canis

\section{Introdução}

As hemoparasitoses são enfermidades de alta morbidade na clínica de pequenos animais e, quando não tratadas, levam à morte do hospedeiro. Nos cães, os hemoparasitos comumente transmitidos por carrapatos são a Ehrlichia canis, Anaplasma platys, Mycoplasma haemocanis, Babesia canis e Hepatozoon canis.

A hepatozoonose canina é uma doença parasitária, causada pelo Hepatozoon spp., que pertence ao filo Protozoa, subfilo Apicomplexa, família Hepatozoidae, subordem Adeleorina (MATHEW et al., 2000).

O cão se infecta ao ingerir carrapatos contendo oocistos maduros na sua hemocele (O'DWYER, 2011). Em seguida, o Hepatozoon spp. parasita leucócitos, tecidos musculares, órgãos parenquimatosos, medula óssea e pleura (BANETH; WEIGLER, 1997), o que promove uma grande diversidade de achados clínicos-laboratoriais, sendo facilmente confundida com outras doenças infecciosas (AGUIAR et al., 2004) e tornando-a de difícil diagnóstico clínico. Esse fato é bastante preocupante, pois esta doença é de caráter debilitante, devido à extensa lesão tecidual em vários órgãos (BANETH; WEIGLER, 1997), e imunossupressora, pois os neutrófilos parasitados são deficientes da mieloperoxidase, tornando-os mais suscetíveis a infecções secundárias (IBRAHIM et al., 1989).

No Brasil, a hepatozoonose canina já foi relatada na região Sudeste (O’DWYER et al., 2001; MUNDIM et al., 2008a; SPOLIDORIO et al., 2009), Centro-Oeste (MUNDIM et al., 2008b; PALUDO et al., 2003; RAMOS et al., 2015) e Sul (LASTA et al., 2009). No Nordeste brasileiro, a prevalência da doença foi estudada apenas no Recife, estado de Pernambuco, por Ramos et al. (2010) e, recentemente, por Rotondano et al. (2015) em Patos, Paraíba.
Diante do exposto, o trabalho teve como objetivo determinar a prevalência da hepatozoonose canina no município de Areia, Paraíba, Brasil.

\section{Material e Métodos}

A pesquisa foi realizada com 151 cães da área urbana do município de Areia (6 ${ }^{\circ} 57^{\prime} 52$ "S e $35^{\circ} 41^{\prime} 53^{\prime}$ W), localizado no estado da Paraíba, região Nordeste do Brasil. Esse município possui área territorial de $266,60 \mathrm{~km}^{2}$ e 23.288 habitantes (IBGE, 2014).

Este estudo baseou-se na avaliação da prevalência da hepatozoonose canina, abrangendo amostragens de todos os bairros do município, quais sejam, Centro, Jussara, Frei Damião, Pedro Perazzo e Cidade Universitária. $\mathrm{O}$ número amostral obtido foi baseado na frequência de $11 \%$ de caninos diagnosticados positivamente para hemoparasitos em um município do estado da Paraíba (SOUSA et al., 2010), com um intervalo de confiança de $95 \%$ e precisão absoluta de 5\% (THRUSFIELD, 2004).

O projeto foi enviado ao Comitê de Ética para Utilização Animal da Universidade Federal da Paraíba e aprovado, estando registrado sob protocolo no ${ }^{0}$ 107/13. Os proprietários foram informados sobre os objetivos do projeto e, caso aceitassem participar, assinavam um Termo de Consentimento Livre e Esclarecido. Para cada animal foi feita uma ficha, contendo resenha, anamnese e exame físico.

Para a realização do hemograma foram colhidos 2,0 $\mathrm{mL}$ de sangue circulante através de venopunção jugular. Este sangue foi acondicionado em microtubos tipo "eppendorf" contendo EDTA a 10\%. Para a avaliação morfológica de células sanguíneas, foi confeccionada uma extensão com sangue e corada com corante rápido, Panótico. $\mathrm{O}$ volume globular, a hematimetria, leucometria global e hemoglobinemia foram determinados utilizando-se o contador de células 
sanguíneas para uso veterinário CC-530 (CELM. Barueri, SP). Os índices hematimétricos de Wintrobe foram determinados por meio de cálculos matemáticos (KERR, 2003).

A pesquisa por hemoparasitos foi realizada por miscroscopia óptica (objetiva de 40X) em extensões sanguíneas de sangue periférico (ponta de orelha) coradas com Panótico. O sangue foi obtido, com a utilização de uma lanceta na face interna da ponta da orelha, após tricotomia e assepsia com álcool 70\%. As amostras sanguíneas foram processadas no Laboratório de Patologia Clínica do Hospital Veterinário do Centro de Ciências Agrárias, Universidade Federal da Paraíba.

Foram avaliadas as fichas de exame clínico e hemograma dos cães positivos, com a finalidade de caracterizar as principais alterações clínicoepidemiológicas. Além disso, foi determinado o grau de parasitemia segundo uma classificação já descrita (MUNDIM et al., 2008b).

\section{Resultados}

Dos 151 cães estudados, 14 (9,3\%) foram positivos para $H$. canis, a partir da visualização de gamontes parasitando neutrófilos. De acordo com a distribuição de animais infectados por bairros, observou-se a maior prevalência no bairro Pedro Perazzo (18,2\%), seguido do Jussara (10,7\%), Frei Damião (7,7\%), Centro (6,9\%) e Cidade Universitária (2,9\%).

As alterações hematológicas observadas em cães portadores de $H$. canis foram diferentes entre os animais, não apresentando padrão de resposta à infecção (Tabela 1).

Os cães apresentaram graus variados de parasitemia, quatro animais $(28,6 \%)$ apresentaram grau leve, sete (50\%), moderado e três $(21,4 \%)$, intenso. Entre os cães positivos, $50 \%$ apresentaram a forma subclínica da doença e os outros 50\% apresentaram sinais clínicos inespecíficos (Tabela 2).

Dos 14 cães diagnosticados com H. canis, 10 $(71,4 \%)$ eram machos e quatro $(28,6 \%)$ fêmeas. Os cães adultos foram os mais acometidos, no total de 11 cães $(78,6 \%)$, e apenas três com idade inferior a um ano
$(21,4 \%)$. Foram mais acometidos cães sem raça definida (SRD), num total de 13 (92,9\%), e apenas um Labrador $(7,1 \%)$. Em relação aos fatores predisponentes, seis animais tinham acesso à rua $(42,9 \%)$ e nove animais $(64,3 \%)$ apresentaram puliciose ou ixodidiose no momento do exame clínico.

TABELA 1: Alterações hematológicas apresentadas pelos 14 cães diagnosticados com Hepatozoon canis no município de Areia, Paraíba.

\begin{tabular}{ccc}
\hline Alterações & N & Porcentagem \% \\
\hline Anemia & 2 & 14,3 \\
Leucocitose & 1 & 7,1 \\
Leucopenia & 2 & 14,3 \\
Neutropenia & 3 & 21,4 \\
Linfopenia & 1 & 7,1 \\
Eosinofilia & 8 & 57,1 \\
Eosinófilos bastonetes & 11 & 78,6 \\
Basofilia & 5 & 35,7 \\
Hiperproteinemia & 11 & 78,6 \\
Hiperfibrinogenemia & 4 & 28,6 \\
\hline
\end{tabular}

$\mathrm{N}$ : número de animais que apresentaram alterações.

TABELA 2: Sinais clínicos observados nos 14 cães diagnosticados com Hepatozoon canis no município de Areia, Paraíba.

\begin{tabular}{ccc}
\hline Sinais clínicos & N & Porcentagem \% \\
\hline Hipertermia & 7 & 50 \\
Apatia & 2 & 14,3 \\
Perda de peso & 2 & 14,3 \\
Hematoquezia & 1 & 7,1 \\
Hiporexia & 1 & 7,1 \\
\hline
\end{tabular}

$\mathrm{N}$ : número de animais que apresentaram alterações.

\section{Discussão}

A prevalência da hepatozoonose canina observada no estudo é considerada alta quando comparada com a prevalência de apenas $0,49 \%$ relatada por Ramos et al. (2010) em Recife e com a ausência de positividade relatada por Rotondano et al. (2015) em cães investigados no município de Patos.

Dessa forma, o presente estudo é o primeiro a relatar a positividade da hepatozoonose canina no 
estado da Paraíba e seus resultados servem como alerta ao clínico veterinário de que a doença está presente na região. Em virtude disso, aconselha-se que mais pesquisas sejam realizadas em outros municípios do Nordeste brasileiro.

Em todos os bairros do município foram observados cães positivos para a hepatozoonose, mas vale ressaltar que o maior índice de positividade para a doença foi observado no bairro Pedro Perazzo, o que pode estar relacionado ao baixo poder aquisitivo dos moradores, cujos animais têm acesso livre à rua, havendo relatos de grande contaminação ambiental por ixodídeos.

No total, foram observados onze tipos de alterações no quadro hematológico que podem estar relacionadas à reação inflamatória tecidual promovida pela doença. Essa variedade mostra que não há um padrão no hemograma de cães infectados com $H$. canis. Além disso, nossos resultados mostram que o animal infectado também pode apresentar o hemograma sem anormalidades, pois onze dos cães avaliados apresentaram a leucometria global dentro dos valores de referência e apenas dois animais apresentaram anemia. A eosinofilia e a presença de eosinófilos bastonetes nos cães sugerem um aumento na demanda desse tipo leucocitário, podendo estar estritamente relacionado a uma resposta ao parasitismo pelo H. canis. Todavia, a resposta contra outros parasitos e a alérgenos não foram descartados no estudo.

Os resultados mostraram que cães infectados com H. canis apresentaram alterações hematológicas e sinais clínicos inespecíficos, este fato está relacionado à baixa patogenicidade do parasita (BANETH; WEIGLER, 1997) por adaptação aos hospedeiros caninos, o que produziria, em muitos casos, uma doença subclínica (BANETH et al., 2003).

A ausência de positividade para outras hemoparasitoses, como Babesia spp. e Ehrlichia canis, pode estar relacionada ao fato de o estudo ser conduzido com cães aparentemente saudáveis e pela pesquisa direta em extensões sanguíneas, diferentemente do constatado por outros autores em trabalhos realizados com cães atendidos na rotina clínica de Hospitais Veterinários e utilizando técnicas moleculares (RAMOS et al., 2010; ROTONDANO et al., 2015). Tendo-se em vista que estas últimas apresentam alta sensibilidade e especificidade, enquanto que a pesquisa direta em esfregaços sanguíneos também possui alta especificidade, porém baixa sensibilidade, outros estudos devem ser realizados, empregando técnicas mais sensíveis, com intuito de reduzir o número de falsos negativos.

Vale ressaltar que no momento do exame clínico, nenhum dos cães positivos apresentou quadro clínico agudo e todos os sinais clínicos apresentados estão de acordo com as observações já relatadas na literatura (BANETH; WEIGLER, 1997; MUNDIM et al., 2008a) e se correlacionam com as lesões promovidas pelo $H$. canis no hospedeiro. Sabendo-se que, após a infecção, os esporozoítos penetram na parede intestinal do hospedeiro (O’DWYER, 2011), é possível observar hematoquezia em infecções recentes, enquanto que os demais sinais clínicos podem estar relacionados a uma infecção de caráter crônico e debilitante.

Estes dados contribuem para a construção do perfil epidemiológico das hemoparasitoses em cães na região Nordeste, além de corroborar com as orientações passadas aos proprietários de cães de estimação, no sentido de não permitirem o livre acesso de seus animais à rua e realizarem o controle de carrapatos, uma vez que a ingestão de carrapatos infectados leva ao desenvolvimento da doença.

Em virtude da alta prevalência encontrada, aconselha-se que o clínico solicite a pesquisa por hemoparasitos em esfregaços sanguíneos quando se deparar com cães apresentando alterações hematológicas e sinais clínicos inespecíficos. Este estudo mostra a necessidade de estudos epidemiológicos contínuos para a detecção de áreas endêmicas, uma vez que se trata de uma doença de difícil diagnóstico clínico.

\section{Agradecimentos}

Os autores agradecem ao Conselho de Desenvolvimento Científico e Tecnológico do Brasil pela concessão de bolsas, às professoras Dra. Ivia Carmem Talieri, Dra. Katerin Elena Bohorquez Grondona, Dra. Valeska Shelda Pessoa de Melo, pertencentes ao Departamento de Ciências Veterinárias, UFPB, à técnica 
Maria de Lourdes Marques de Souza, do Laboratório de Patologia Clínica, UFPB, e aos alunos voluntários do curso de Medicina Veterinária, UFPB, que participaram durante a etapa de colheita de amostras sanguíneas para realização desta pesquisa.

\section{Referências}

AGUIAR, D. M.; RIBEIRO, M. G.; SILVA, W. B.; DIAS JR, J. G.; MEGID, J.; PAES, A. C. Hepatozoonose canina: achados clínicoepidemiológicos em três casos. Arquivo Brasileiro de Medicina Veterinária e Zootecnia, Belo Horizonte, v. 56, n. 3, p. 411-413, 2004.

BANETH, G.; MATHEW, J. S.; SHKAP, V.; MACINTIRE, D. K.; BARTA, J. R.; EWING, S. A. Canine hepatozoonosis: two disease syndromes caused by separate Hepatozoon spp. Trends in Parasitology, Cambridge, v. 19, n. 1, p. 27-31, 2003.

BANETH, G.; WEIGLER, B. Retrospective case-control study of hepatozoonosis in dogs in Israel. Journal of Veterinary Internal Medicine, Lakewood, v. 11, n. 6, p. 365-370, 1997.

IBGE - Instituto Brasileiro de Geografia e Estatística. 2014. Cidades do Brasil. Disponível em: <http://cod.ibge.gov.br/2368Z>. Acesso em: 25 ago. 2015.

IBRAHIM, N. D. G.; RAHAMATHULLA, P. M.; NJOKU, C. O. Neutrophil myeloperoxidase deficiency associated with canine hepatozoonosis. International Journal for Parasitology, Amsterdam, v. 19, n. 8, p. 915-918, 1989.

KERR, M. G. Exames laboratoriais em medicina veterinária: bioquímica clínica e hematologia. 2. ed. São Paulo: Roca, 2003. $436 \mathrm{p}$.

LASTA, C. S.; SANTOS, A. P.; MELLO, F. P. S.; LACERDA, L. A.; MESSICK, J. B.; GONZÁLES, F. H. D. Infecção por Hepatozoon canis em canino doméstico na região Sul do Brasil confirmada por técnicas moleculares. Ciência Rural, Santa Maria, v. 39, n. 7, p. 2135-40, 2009.

MATHEW, R. A.; BUSSCHE, V. D.; EWING, S. A.; MALAYER, J. R.; LATHA, B. R.; PANCIERA, R. J. Phylogenetic relationships of Hepatozoon (Apicomplexa: Adeleorina) based on molecular, morphologic, and life-cycle characters. Journal of Parasitology, Lawrence, v. 86, n. 2, p. 366-372, 2000.

MUNDIM, A. V.; MORAIS, I. A.; TAVARES, M.; CURY, M. C.; MUNDIM, M. J. Clinical and hematological signs associated with dogs naturally infected by Hepatozoon sp. and with other hematozoa: a retrospective study in Uberlândia, Minas Gerais, Brazil. Veterinary Parasitology, Amsterdam, v. 153, n. 1-2, p. 3-8, 2008a.
MUNDIM, E. C. S.; FRANCISCO, M. M. S.; SOUZA, J. N.; ALENCAR, M. A. G.; RAMALHO, P. C. D. Incidência de hemoparasitoses em cães (Canis familiares) de rua capturados pelo Centro de Controle de Zoonoses (CCZ) da cidade de ANÁPOLISGO. Ensaios e Ciência: Ciências Biológicas, Agrárias e da Saúde, Valinhos, v. 12, n. 2, p. 107-115, 2008 b.

O'DWYER, L. H. Brazilian canine hepatozoonosis. Revista Brasileira de Parasitologia Veterinária, Jaboticabal, v. 20, n. 3, p. 181-193, 2011.

O'DWYER, L. H.; MASSARD, C. L.; SOUZA, J. C. P. Hepatozoon canis infection associated with dog ticks of rural areas of Rio de Janeiro State, Brazil. Veterinary Parasitology, Amsterdam, v. 94, n. 3, p. 143-150, 2001

PALUDO, G. R.; DELL'PORTO, A.; CASTRO E TRINDADE, A. R.; MCMANUS, C.; FRIEDMAN, H. Hepatozoon spp.: report of some cases in dogs in Brasília, Brazil. Veterinary Parasitology, Amsterdam, v. 118, n. 3-4, p. 243-248, 2003.

RAMOS, C. A. N.; BABO-TERRA, V. J.; PEDROSO, T. C.; SOUZA FILHO, A. F.; ARAÚJO, F. R.; CLEVELAND, H. P. K. Molecular identification of Hepatozoon canis in dogs from Campo Grande, Mato Grosso do Sul, Brazil. Revista Brasileira de Parasitologia Veterinária, Jaboticabal, v. 24, n. 2, 2015.

RAMOS, R.; RAMOS, C.; ARAÚJO, F.; OLIVEIRA, R.; SOUZA, I.; PIMENTEL, D.; GALINDO, M.; SANTANA, M.; ROSAS, E.; FAUSTINO, M.; ALVES, L. Molecular survey and genetic characterization of tick-borne pathogens in dogs in metropolitan Recife (north-eastern Brazil). Parasitology Research, Heidelberg, v. 107, p. 1115-1120, 2010.

ROTONDANO, T. E. F.; ALMEIDA, H. K. A.; KRAWCZAK, F. S.; SANTANA, V. L.; VIDAL, I. F.; LABRUNA, M. B.; AZEVEDO, S. S.; ALMEIDA, A. M. P.; MELO, M. A. Survey of Ehrlichia canis, Babesia spp. and Hepatozoon spp. in dogs from a semiarid region of Brazil. Brazilian Journal of Veterinary Parasitology, Jaboticabal, v. 24, n. 1, p. 52-58, 2015.

SPOLIDORIO, M. G.; LABRUNA, M. B.; ZAGO, A. M.; DONATELE, D. M.; CALIARI, K. M.; YOSHINARI, N. H. Hepatozoon canis infecting dogs in the State of Espírito Santo, southeastern Brazil. Veterinary Parasitology, Amsterdam, v. 163, n. 4, p. 357-61, 2009.

SOUSA, M. I. L.; SOUZA, A. P.; MOREIRA, M. N.; FEITOSA, T. F.; DIAS, R. A.; ISIDÓRIO, U. A. Aspectos epidemiológicos das hemoparasitoses caninas ocorridas no ano de 2009 no Hospital veterinário da Universidade Federal de Campina Grande, Patos PB. In: REUNIÃO ANUAL DA SBPC, 62, 2010, Natal. Resumos... Natal: SBPC, 2010. Versão eletrônica.

THRUSFIELD, M. Epidemiologia veterinária. 2. ed. São Paulo: Roca, 2004. 556 p. 